\title{
Nuclei of aged myofibres undergo structural and functional changes suggesting impairment in RNA processing
}

\author{
M. Malatesta, ${ }^{1}$ F. Perdoni, ${ }^{2}$ S. Muller, ${ }^{3}$ C. Zancanaro, ${ }^{1}$ C. Pellicciari ${ }^{2}$ \\ ${ }^{1}$ Dipartimento di Scienze Morfologico-Biomediche, Sezione di Anatomia e Istologia, University of Verona, \\ Italy; ${ }^{2}$ Laboratorio di Biologia Cellulare, Dipartimento di Biologia Animale, University of Pavia, Pavia, \\ Italy; ${ }^{3} \mathrm{CNRS}$, Institut de Biologie Moléculaire et Cellulaire, Immunologie et Chimie Thérapeutiques, \\ Strasbourg, France
}

(C)2009 European Journal of Histochemistry

Advancing adult age is associated with a progressive decrease in skeletal muscle mass, strength and quality known as sarcopenia. The mechanisms underlying age-related skeletal muscle wasting and weakness are manifold and still remain to be fully elucidated. Despite the increasing evidence that the progress of muscle diseases leading to muscle atrophy/dystrophy may be related to defective RNA processing, no data on the morpho-functional features of skeletal muscle nuclei in sarcopenia are available at present. In this view, we have investigated, by combining morphometry and immunocytochemistry at light and electron microscopy, the fine structure of myonuclei as well as the distribution and amount of RNA processing factors in skeletal myofibres of biceps brachii and quadriceps femoris from adult and old rats. Results demonstrate that the myonuclei of aged type II fibres show an increased amount of condensed chromatin and lower amounts of phosphorylated polymerase II and DNA/RNA hybrid molecules, clearly indicating a decrease in pre-mRNA transcription rate compared to adult animals. In addition, myonuclei of aged fibres show decreased amounts of nucleoplasmic splicing factors and an accumulation of cleavage factors, polyadenilated RNA and perichromatin granules, suggesting a reduction in the processing and transport rate of premRNA. During ageing, it seems therefore that in rat myonuclei the entire production chain of mRNA, from synthesis to cytoplasmic export, is less efficient. This failure likely contributes to the reduced responsiveness of muscle cells to anabolic stimuli in the elderly.

Key words: ageing, sarcopenia, skeletal muscle, cell nucleus, transcription, splicing.

Correspondence: Manuela Malatesta

Dipartimento di Scienze Morfologico-Biomediche, Sezione di Anatomia e Istologia, Università degli Studi di Verona,

Strada Le Grazie, 837134 Verona, Italy

Tel.: +39.045.8027155.

Fax: +39.045.8027163.

E-mail: manuela.malatesta@univr.it

Paper accepted on 28 May 2009

European Journal of Histochemistry

2009; vol. 53 issue 2 (April-June): 97-106
A dvancing adult age is associated with a progressive decrease in skeletal muscle mass, strength and quality known as sarcopenia (reviews in Roubenoff et al., 2000; Doherty, 2003; Deschenes, 2004). Sarcopenia affects healthy, physically active subjects and the rate of muscle loss in humans has been estimated to range 1-2\% per year past the age of 50 (Hughes et al., 2002). Therefore, sarcopenia represents a powerful risk factor for frailty, loss of independence and physical disability in elderly since it is associated with decreased functional performance, greater risk of falls, increased motor disability. Moreover, the reduction in muscle mass can be associated with osteoporosis (Szulc et al., 2005), altered thermogenic capacity (Wilson and Morley, 2003), insulin resistance, type 2 diabetes mellitus, dyslipidemia, and hypertension (Karakelides and Sreekumaran Nair, 2005). Understanding the mechanisms leading to sarcopenia represents therefore an essential step to prevent and combat illness and disability at advanced age.

The mechanisms underlying age-related skeletal muscle wasting and weakness are manifold and still remain to be fully elucidated (review in Ryall, 2008; Thompson, 2009); they may include denervation and reinnervation of motor units (Ansved and Larsson, 1989), decline in anabolic hormone concentrations (Tenover, 1997), increased concentrations of inflammatory mediators (Bruunsgaard et al., 2001), decrement in microvascular function and exercise tolerance (Payne and Bearden, 2006), impairment of proteolytic and autophagic pathways (Combaret et al., 2009), loss of satellite cells (Verdijk et al., 2007), depletion of myonuclei (Dirks and Leeuwenburgh, 2005).

Despite the extensive literature on sarcopenia and, in particular, on the debated role of myonuclei loss in fibre atrophy (Dirks and Leeuwenburgh, 2005, Dupont-Versteegden, 2005; Gundersen and 
Bruusgaard, 2008), at present no data on the morpho-functional features of skeletal muscle nuclei in sarcopenia are available.

In eukaryotic cells, nuclear ribonucleoprotein (RNP)-containing structures are part of the transcription and splicing machinery. At electron microscopy, these structures are recognized as perichromatin fibrils (PF), perichromatin granules (PG), and interchromatin granules (IG) (Fakan, 2004; Spector, 1996). PF are the morphological equivalent of hnRNA transcription and co-transcriptional splicing, while IG represent a storage site for snRNP and non-snRNP splicing factors, and $P G$ are involved in the storage and the nucleus-to-cytoplasm transport of mRNA (recently reviewed in Biggiogera et al., 2007). All these components have specific intranuclear location: $P F$ and $P G$ are at the periphery of condensed chromatin, and IG in the so-called interchromatin space (for a review, see Puvion and PuvionDutilleul, 1996). This is a necessary prerequisite for the maturation of nuclear RNAs, so that whenever transcription and/or splicing are altered, the organization, composition, and intranuclear location of RNP-containing structures are also affected (Biggiogera et al., 2004, 2007).

In this view, in the present study, by combining morphometry and immunocytochemistry at light and electron microscopy, we examined the fine structure of myonuclei as well as the distribution and amount of factors involved in RNA processing in skeletal myofibres of adult and old rats. As study system, we chose the biceps brachii and the quadriceps femoris muscles, which mostly contain fast type II fibres and are therefore largely affected by sarcopenia (Larsson et al., 1978; Shorey et al., 1998; Lexell, 1995).

\section{Materials and Methods}

Two adult (9 months of age) and two old (28 months of age) male Wistar rats were used. All animals were bred under controlled environmental conditions with a $12 \mathrm{~h}$ light/dark cycle, and fed ad libitum with a standard commercial chow. The experimental protocols comply with the guidelines of the Italian Ministry of Health as well as with internationally recognized guidelines. The rats were deeply anaesthetised with pentobarbital (50 $\mathrm{mg} / \mathrm{Kg}$ i.p.) and then perfused via the ascending aorta with a brief prewash of $0.09 \% \mathrm{NaCl}$ solution followed by $300 \mathrm{~mL}$ of a fixative solution containing $4 \%$ paraformaldehyde in $0.1 \mathrm{M}$ phosphate buffer, pH 7.4 at $4^{\circ} \mathrm{C}$. Biceps brachii and quadriceps femoris muscles were quickly removed and placed in the same fixation solution for $2 \mathrm{~h}$ at $4^{\circ} \mathrm{C}$.

\section{Light microscopy}

After fixation, muscle samples were dehydrated with ethanol and embedded in paraffin wax. Five$\mu$ m-thick muscle samples were sectioned both transversally and longitudinally and then submitted to immunohistochemical procedures for fibre typing: sections were incubated overnight at $4^{\circ} \mathrm{C}$ with a mouse monoclonal antibody recognizing the heavy chain of skeletal fast fibre myosin (clone MY-32, Sigma-Aldrich, Buchs, Switzerland), then revealed with an Alexa 488 conjugated antibody against mouse IgG (Molecular Probes, Invitrogen, Milan). The sections were finally counterstained for DNA with $0.1 \mu \mathrm{g} / \mathrm{mL}$ Hoechst 33258 to label the myonuclei and to detect the occurrence of apoptosis based on chromatin morphology. Micrographs were taken in an Olympus BX51 microscope equipped with a $100 \mathrm{~W}$ mercury lamp under the following conditions: $330-$ to $385-\mathrm{nm}$ excitation filter (excf), 400-nm dichroic mirror (dm), and 420-nm barrier filter (bf), for Hoechst 33258; 450- to 480-nm excf, 500-nm dm, and $515 \mathrm{~nm}$ bf for Alexa 488. Images were recorded with an Olympus Camedia C-5050 digital camera and stored on a PC by the Olympus software for processing and printing. Morphometrical evaluations were performed by using the software Image $\mathrm{J}$ (NIH, USA). The cross-sectional area of transversally cut fibres was measured on 100 myofibres per sample at $40 \mathrm{X}$ magnification. Moreover, the percentage of fast and slow muscle fibres was calculated on a total of 300 fibres per muscle. To evaluate nuclear density in fast fibres, the area of longitudinally sectioned myofibres was measured up to a total of about $15,000{\mu m^{2}}^{2}$ per muscle

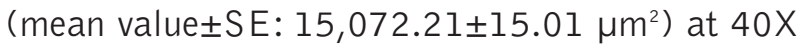
magnification, myonuclei were counted and the nuclear density was expressed as the number of myonuclei/100 $\mu^{2}$ of myofibre area. Finally, the percentage of apoptotic nuclei was evaluated, using a $\times 20$ objective lens, on a total of 25 microscope fields (at least 2000 myonuclei per muscle sample were counted). 


\section{Transmission electron microscopy}

After fixation, muscle samples were washed in Sörensen buffer and subsequently in phosphate buffer saline (PBS), kept in $0.5 \mathrm{M} \mathrm{NH}_{4} \mathrm{Cl}$ in PBS for 45 min to block free aldehydes, dehydrated with ethanol and embedded in LR White resin polymerized under U.V. light. Ultrathin sections were collected on Formvar-carbon coated nickel grids and used for morphometrical and immunocytochemical analyses.

Morphometrical evaluations were made on micrographs $(x 11,000)$ of twenty myonuclei from longitudinally sectioned fibres per muscle by using a computerized image analysis system (AnalySIS Image processing, Soft Imaging System $\mathrm{GmbH}$, Muenster, Germany). The following parameters were considered: area of nuclei, nucleoli and fibrillar centres (FCs); percentage of nuclear area occupied by condensed chromatin; percentage of total FC, dense fibrillar component (DFC) and granular component (GC) area per nucleolus; $P G$ density $\left(P G / \mu m^{2}\right.$ of nucleoplasm); nuclear pore frequency (NP/ $\mu \mathrm{m}$ of perimeter).

To investigate the fine distribution of some RNA transcription and processing factors, muscle sections were treated with the following probes: mouse monoclonal antibodies directed against the active phosphorylated form of RNA polymerase II (Research Diagnostic Inc., Flanders, NJ) or against the $(\mathrm{Sm})$ snRNP (small nuclear RNP) core protein (Abcam, Cambridge, MA); rabbit polyclonal antibodies were used against DNA/RNA hybrid molecules (Testillano et al., 1994) specifically occurring in the transcriptional sites (Malatesta et al., 2008, 2009), and the cleavage stimulation factor CstF (Veraldi et al., 2001). Sections were incubated with the primary antibodies revealed by secondary gold-conjugated probes as described in Malatesta et al. (2009). As controls, some grids were incubated without the primary antibody and then processed as described above.

In situ hybridization for polyadenylated RNAs was carried out by using a biotinylated oligo $d(T)$ (Sigma-Aldrich, Buchs, Switzerland) reacted with a gold-conjugated goat anti-biotin antibody (Aurion, Wageningen, The Netherlands) as reported in Cisterna et al. (2008). As controls, some grids were incubated in the absence of the probe, or pre-treated with $0.1 \%$ RNAase for $18 \mathrm{~h}$.

To reduce chromatin contrast and selectively reveal nuclear RNP constituents, the sections were bleached by the EDTA method (Bernhard, 1969) and observed in a Philips Morgagni TEM operating at $80 \mathrm{kV}$ and equipped with a Megaview II camera for digital image acquisition.

Quantitative assessment of the immunolabelling was carried out by estimating the gold grain density over selected cellular compartments on sections treated in the same run. The surface area of nucleoplasm and nucleolus was measured on fifteen randomly selected electron micrographs $(x 22,000)$ from each animal by using a computerised image analysis system (AnalySIS Image processing, Soft Imaging System GmbH). For background evaluation samples treated in the absence of primary antibody or pre-treated with RN Aase were considered. Gold grains present over each selected compartment were counted and the labelling density was expressed as number of gold grains $/ \mu m^{2}$.

\section{Statistics}

Results for each measured parameter were pooled according to the experimental groups and the meanststandard error of the mean (SE) values calculated. Statistical comparisons were performed by the Mann Whitney $U$ test (significance set at $p \leq 0.05$ ).

\section{Results}

\section{Light microscopy}

Morphometrical results are summarised in Figure 1. Fast myofibres (Figure 2 a-d) showed markedly smaller cross-sectional area in both biceps and quadriceps muscles of old rats compared to adult animals; slow fibres also were reduced in size in old rats, but the difference between the two groups was not statistically significant. The percentage of slow fibres was low in both muscles, as expected. In old rats the percentage of slow fibre was higher than in adult animals, but the difference was not significant. Nuclear density in the fast fibres (Figure $3 a, b, f$ ) was significantly higher in old than in adult rats, in both biceps and quadriceps muscles. Morphologically recognizable apoptotic nuclei (Figure $3 \mathrm{c}$-e) were quite scarce (generally less than $0.2 \%$ ) and their percentage was similar in adult and old animals in both biceps and quadriceps muscles. 


\section{Transmission electron microscopy}

In all muscle samples myofibres contained multiple, elongated cell nuclei typically placed close to the sarcolemma. Myonuclei generally showed finely irregular border, condensed chromatin clumps at both the nuclear and nucleolar periphery, one roundish nucleolus characterized by a few FC, and abundant DFC and GC (Figure 4). In the nucleoplasm, all the usual RNP structural constituents involved in pre-mRNA transcription and processing - i.e., PF, PG and IG - were obvious. Morphological evidence of nuclear apoptosis was never observed in all muscle samples examined.

In both biceps and quadriceps muscles of old rats, myonuclei had smaller area and were characterized by a higher percentage of condensed chromatin, a higher density of $P G$ and nucleoli with
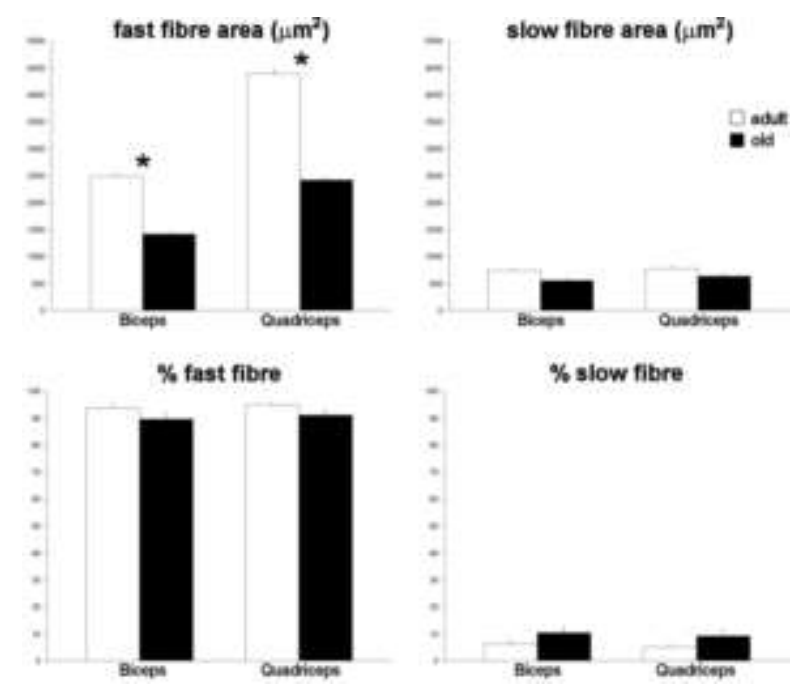

Figure 1. Light microscopy morphometric parameters considered in biceps and quadriceps muscle myofibres of adult and old rats (means $\pm S E)$. Asterisks indicate significantly different values.

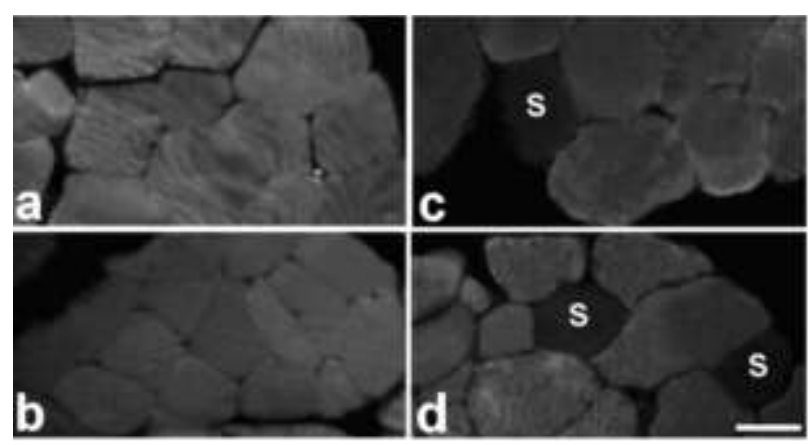

Figure 2. Immunolabelled fast type II fibres in the biceps $(a, b)$ and quadriceps (c, d) of adult (a, c) and old (b, d) rats. Slow (S) type I fibres are scarce. Note the smaller size of fast type II fibres in old animals. Bar: $30 \mu \mathrm{m}$.
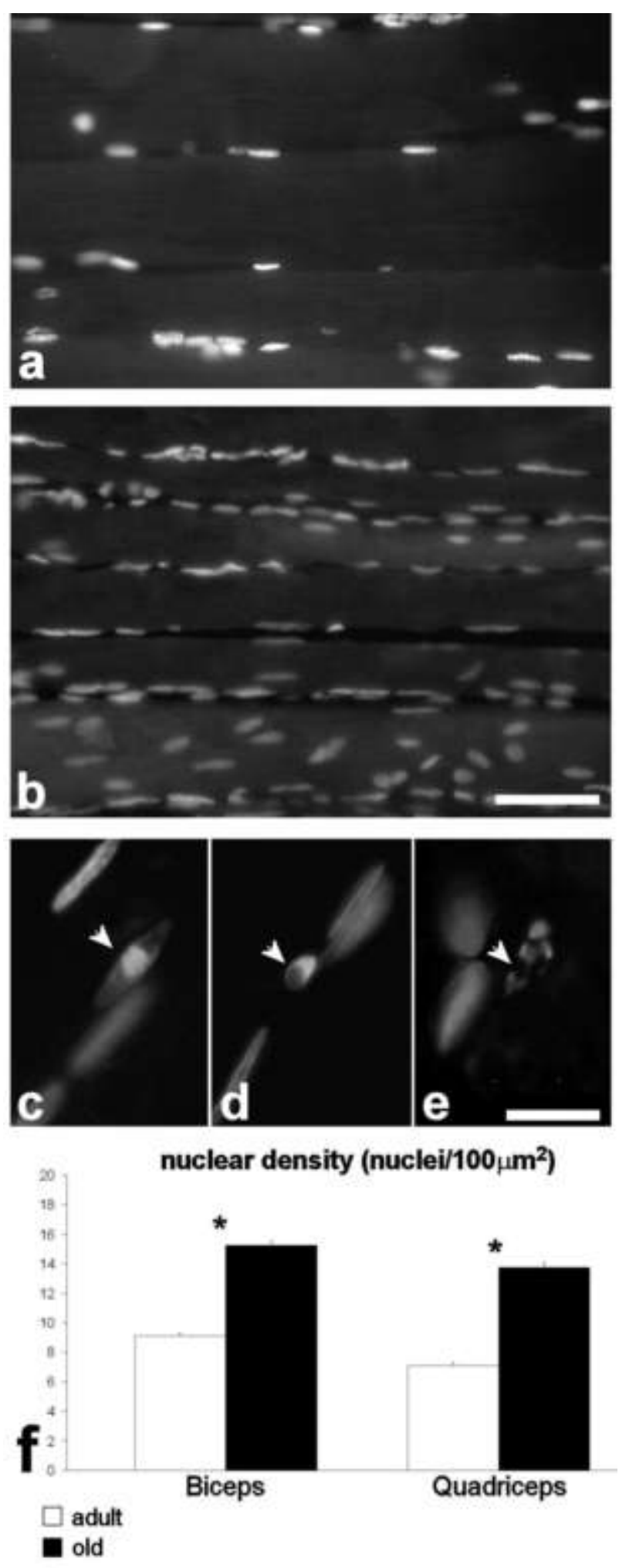

Figure 3. Quadriceps muscle from an adult (a) and an old (b) rat; DNA was stained with Hoechst 33258. Bar: $50 \mu \mathrm{m}$. Very few apoptotic nuclei with typical chromatin condensation and nuclear fragmentation (arrows) were observed in both adult (c,d) and old (e) animals. Bar: $20 \mu \mathrm{m}$. The nuclear density is higher in both biceps and quadriceps muscles of old animals (f); asterisks show significantly different values. 
smaller FC compared to adult animals (Figure 5). Conversely, there was no difference in either the nucleolar area or the percentage of DFC and GC in adult and old rats.

Immunocytochemical results showed similar distribution of polymerase II, DNA/RNA hybrid molecules, snRNPs, cleavage factor CstF and polyadenylated RNA in adult and old rats. In both animal groups, polymerase II was exclusively associated with PF (Figure $6 a, b$ ); DNA/RNA hybrid molecules specifically labelled PF (Figure $6 a, b$ ) and the nucleolar DFC (not shown); snRNPs were restricted to PF and, at a lesser degree, to IGs (Figure $6 \mathrm{c}, \mathrm{d}$ ); CstF and polyadenylated RNA were located essentially on the PF and also on RN $P$ tails rising from $P G$ (Figure $6 \mathrm{e}-\mathrm{h}$ ).

Quantitative evaluation of the immunolabelling (Figure 7) revealed lower nucleoplasmic density of polymerase II, DNA/RNA hybrid molecules and snRNPs in both biceps and quadriceps muscles of old rats; conversely, CStF and polyadenylated RNA were more abundant in the nucleoplasm of old than adult rats. No difference was found between the two age groups for nucleolar labelling obtained with any probes.

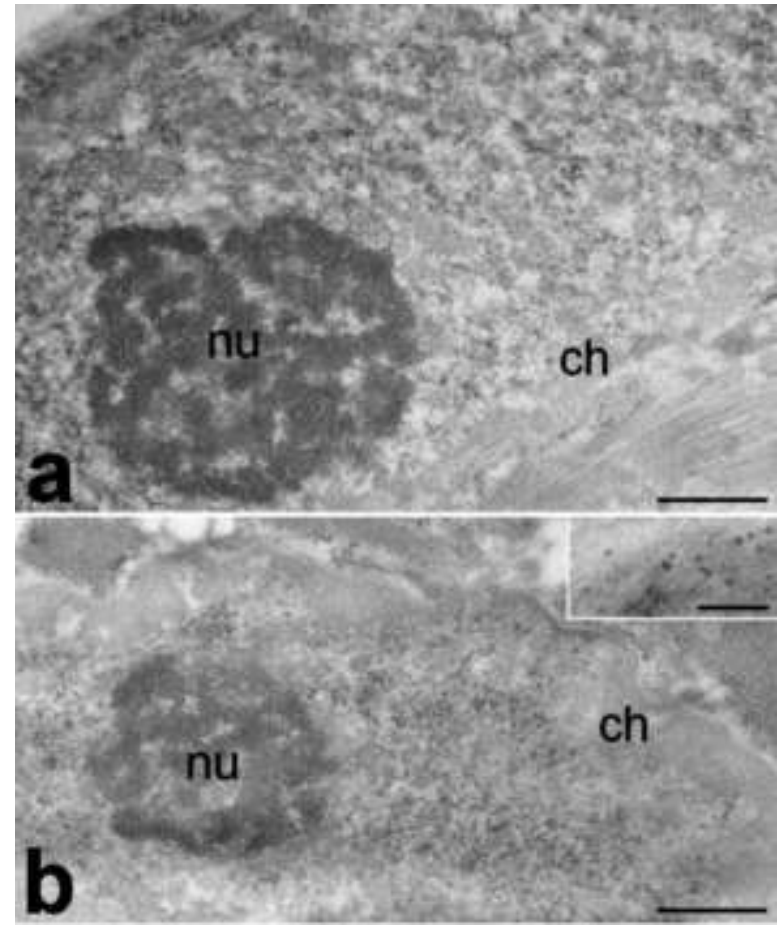

Figure 4. Myonuclei of quadriceps from adult (a) and old (b) rats. In old animals, condensed chromatin (ch) is more abundant and perichromatin granules are more numerous (inset) than in adult ones, whereas nucleoli (nu) show a similar aspect. Bars: 500 nm; inset $250 \mathrm{~nm}$.
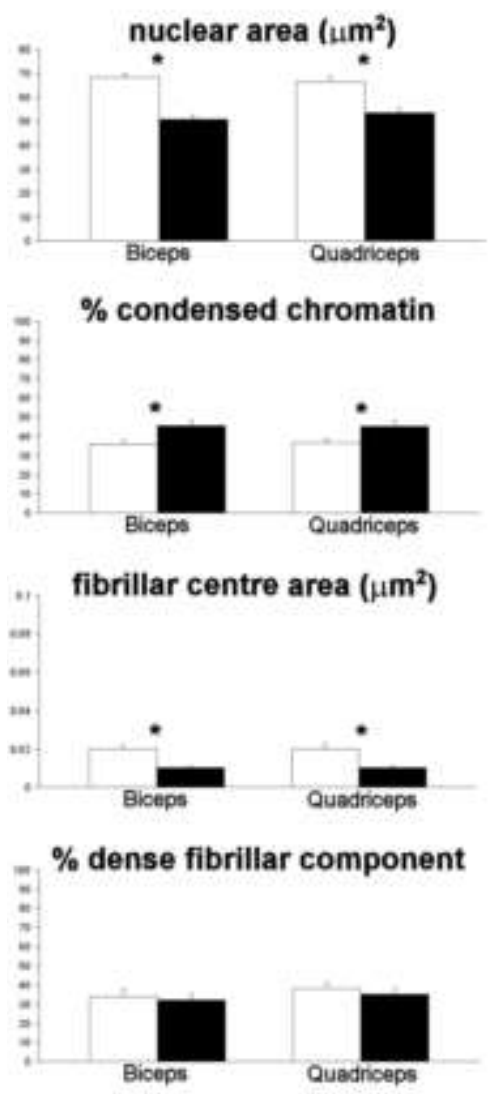

nucleolar area $\left(\mu \mathrm{m}^{2}\right)$
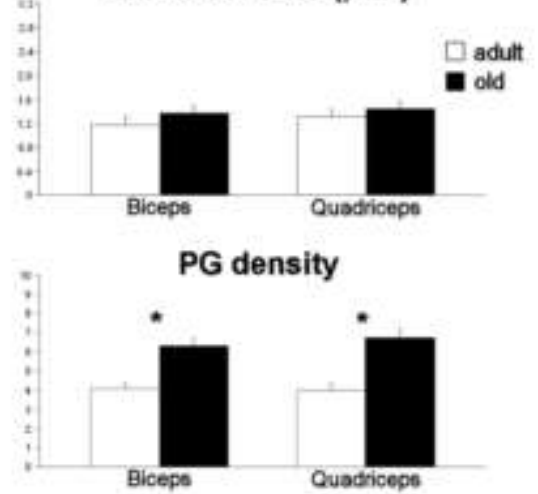

$\%$ fibrillar centre

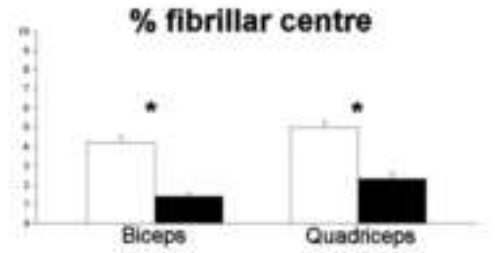

$\%$ granular component

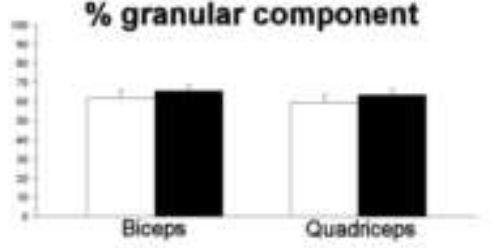

Figure 5. Electron microscopy morphometric parameters considered in myonuclei of biceps and quadriceps muscles of adult and old rats (means $\pm S E$ ). Asterisks show significantly different values. 

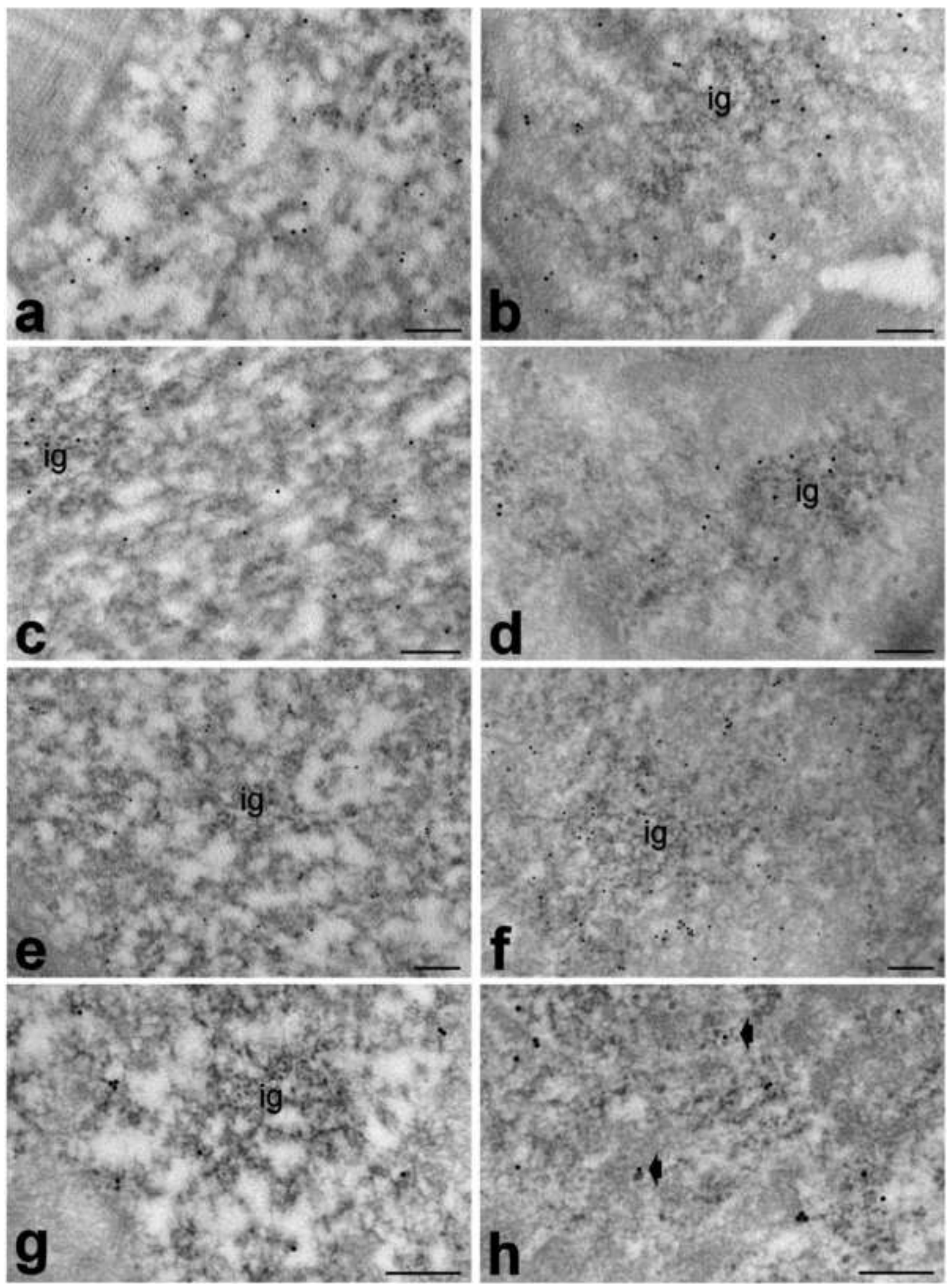

Figure 6. Myonuclei from adult (a,c,e,g) and old (b,d,f,h) rats. a,b. Quadriceps muscles, anti-polymerase II (12 nm) and anti-DNA/RNA hybrid $(5 \mathrm{~nm})$ antibodies: both antibody probes specifically label perichromatin fibrils. c,d. Biceps muscles, anti-snRNP antibody: perichromatin fibrils and interchromatin granules (ig) are labelled. e,f. Biceps muscles, anti-CStF antibody: gold grains specifically label perichromatin fibrils. g,h. Quadriceps muscles, polyadenylated RNA: the labelling occurs on perichromatin fibrils and on RNP tails rising from perichromatin granules (arrows). Bars: $250 \mathrm{~nm}$. 

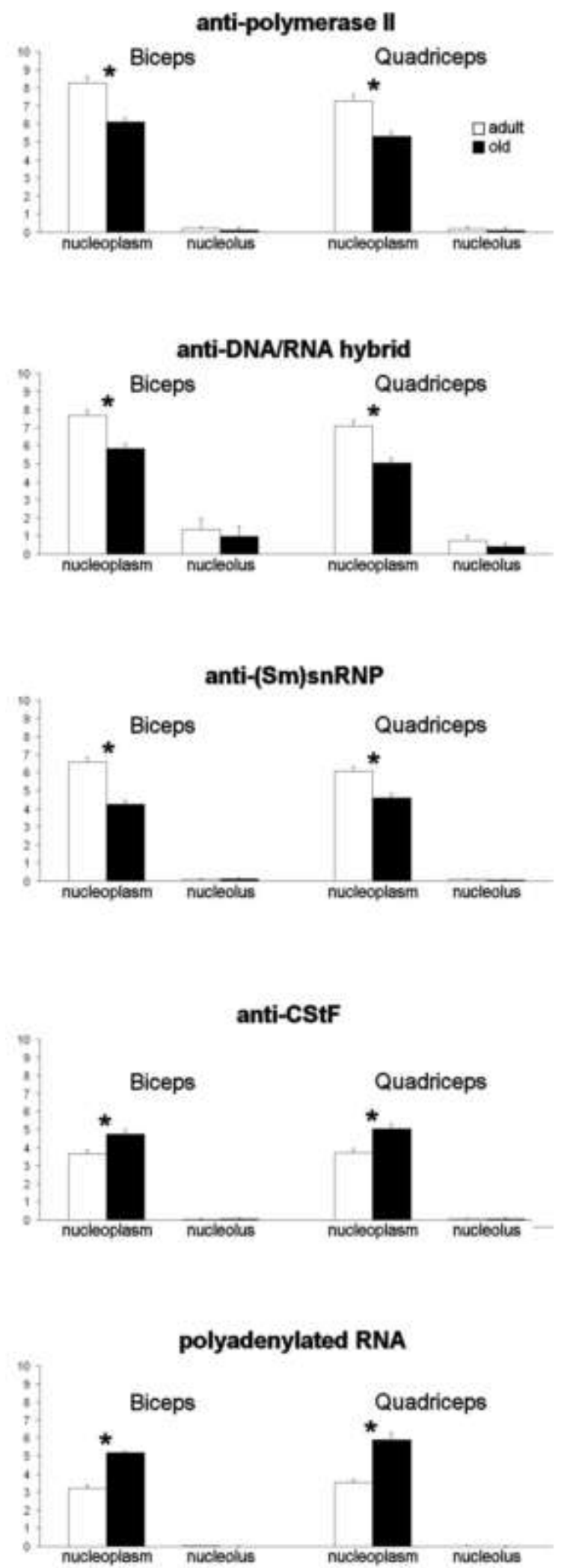

Figure 7. Quantitative immunoelectron microscopy of myonuclei of biceps and quadriceps mucles from adult and old rats; labelling density (gold grains $/ \mu \mathrm{m}^{2}$ ) of several RNA processing factors in the nucleoplasm and nucleolar compartments (means $\pm S E$ ). Asterisks show significantly different values.
Background values were negligible in all immunolabelling experiments (not shown).

\section{Discussion}

Sarcopenia entails reduction of skeletal muscle mass, strength and resistance, with consequent impairment of performance in older individuals. In sarcopenia, muscles undergo loss of myofibres as well as decrease in total cross-sectional area of the remaining myofibres, both phenomena affecting preferentially type II fibres (Larsson et al., 1978; Shorey et al., 1998; Lexell, 1995). Accordingly, our data demonstrate a significant reduction in type II myofibre size in both biceps brachii and quadriceps femoris muscles of old rats. In addition, an increased - although not statistically significant - percentage of type I fibres is found in old animals, suggesting a preferential loss of type II fibres; however, there is conflicting evidence of changes in fibre type proportion in muscle in sarcopenia, some authors reporting no modifications (review in Hepple, 2003).

Muscle fibres are multinucleated syncitia and a reduction in fibre size could provoke alteration in nuclear number, nuclear domain size or both; however, studies on fibre atrophy conflict on whether these features undergo modifications or not. This is mainly due to the differences in muscle, myofibre and atrophy type that were investigated (for a recent review see Brooks et al., 2009); moreover, it has been demonstrated that myofibre size and nuclear number can be uncoupled in different phases of the ageing process (Wada et al., 2003; Bruusgaard et al., 2006). In the present study, it was found that nuclear density is higher in aged type II myofibres, according to previous reports on muscle atrophy (Tomonaga, 1977; Manta et al., 1987; Gallegly et al., 2004; Kadi et al., 2004; Edström and UIfhake, 2005). This finding, together with the scarce presence of morphologically recognizable apoptotic nuclei (both at light and electron microscopy) suggests that only a limited nuclear death rate occurs in ageing skeletal muscles. It has been hypothesized that apoptotic induced loss of myonuclei may be involved in muscle protein degradation, thus contributing to fibre atrophy and, finally, myocyte death (Leeuwenburgh, 2003; Dirks and Leeuwenburgh, 2005; Alway and Siu, 2008). However, the role of apoptosis in myofibre depletion is still debated 
(Dirks and Leeuwenburgh, 2005, DupontVersteegden, 2005), some authors asserting that apoptotic signals mostly originate from satellite cells and connective tissue rather than from myofibres (Gundersen and Bruusgaard, 2008). The present observations are in agreement with previous data on hindlimb muscles of old mice demonstrating that apoptosis is quite scarce in both sedentary and trained animals (Zancanaro et al., 2007) as well as with ultrastructural studies showing the absence of typical apoptosis in atrophic myofibres (Rodrigues and Schmalbruch, 1995; Borisov and Carlson, 2000). However, it should be considered that our data have been collected in old animals where the sarcopenic process had already come to an advanced stage; this implies that a role of apoptosis in earlier phases cannot be excluded.

The myonuclei of aged type II fibres show morpho-functional features suggestive of a reduced activity in comparison to myonuclei of adult individuals. Smaller size and an increased amount of condensed chromatin together with lower quantity of phosphorylated polymerase II and DNA/RNA hybrid molecules, specifically occurring at transcriptional sites, clearly indicate a decrease in pre-mRNA transcription rate (Malatesta et al., 2008, 2009). The biological activity of many transcription factors is modulated by the redox state of the cell (Roijkind et al., 2002) and it is possible that the progressive increase of oxidative stress during ageing (Stadtman, 2004, 2006; Lesnefsky and Hoppel, 2006) contributes to the reduction of the transcription rate. According to the decrease in transcription rate, myonuclei of old rats contain lower amounts of nucleoplasmic splicing factors, involved in the co-transcriptional pre-mRNA splicing (Lührmann et al., 1990). In addition, myonuclei of aged fibres show an accumulation of cleavage factors and polyadenylated RNA, strongly suggesting a reduction in the processing and transport rate of pre-mRNA. The accumulation of $P G$ further supports the hypothesis of decreased pre-mRNA transcriptional/co-transcriptional activities and reduced cytoplasmic export. In fact, $P G$ represent storage and/or transport sites for spliced mRNA, and $P G$ accumulation has been described as a consequence of altered pre-mRNA processing as well as of impaired intranuclear or nucleus-to-cytoplasm transport (e.g. Puvion et al.,
1977; Puvion-Dutilleul et al., 1981; Lafarga et al., 1993; Zancanaro et al., 1993). Accordingly, a decrease in nucleus-to-cytoplasm transport factors (Pujol et al., 2002) has been reported during ageing, when the degradation systems also undergo alterations (Jameson, 2004) leading to the accumulation of crosslinked, insoluble and often oxidized proteins which may damage the intracellular transport mechanisms (Hallen, 2002; Kim et al., 2001); the intranuclear accumulation of RNA with poly $(A)$ tails, known to be involved in stabilization, localization and translation of mRNAs (e.g. Soreq et al., 1974; Marbaix et al., 1975), could be also related to the reduced efficacy of the degradation machinery. Interestingly, malfunctions of the proteolytic and autophagic pathways are thought to be involved in sarcopenia (Attaix et al., 2005; Combaret et al., 2009).

On the other hand, myonuclear nucleoli in aged fibres do not show structural modifications suggestive of a marked activity decline. In fact, a significant reduction was observed in the FC only, whereas minor changes were found for the DFC and GC. However, the slight decrease of the DFC together with the lower (though not statistically significant) amount of DNA/RNA hybrid molecules in the nucleoli in aged fibres points to a reduction in the $r$ RNA synthetic rate (Schwarzacher and Wachtler, 1993; Shaw and Jordan, 1995); in addition, the small increase of the GC may be the consequence of an impaired nucleus-to-cytoplasm transport of pre-ribosomal subunits.

It seems therefore that, during ageing, in myonuclei the entire production chain of RNA, from synthesis to cytoplasmic export, is less efficient. The reduction in muscle tissue mass with ageing has been attributed to a disruption in the regulation of skeletal muscle protein turnover, leading to a structural imbalance between muscle protein synthesis and degradation (reviewed in Koopman, 2009); in this view, altered RNA pathways may contribute to the reduced nuclear responsiveness to cellular metabolic needs. Accordingly, no decrease in the basal rates of muscle protein synthesis has been found in elderly, whereas during ageing the muscle protein synthetic response to the main anabolic stimuli, i.e. food intake and/or physical activity, is blunted (Koopman, 2009). Moreover, a reduction of nuclear activity would be consistent with the reduction in nuclear domain 
size observed in aged fibres.

Interestingly, most of the changes found in myonuclei of aged fibres are reminiscent of those observed in senescent hepatocyte nuclei (Malatesta et al., 2003, 2004), and indicate that impairment in RNA pathways represents a general phenomenon during ageing. Moreover, defective RNA pathways due to nuclear sequestration of pre-mRNAs and processing factors have been recently related to diseases leading to muscle atrophy/dystrophy (Cardani et al., 2006; Wheeler and Thornton, 2007): the results of the present study suggest intriguing similarities in the basic mechanisms of sarcopenic and dystrophic processes, thus opening promising perspectives for preventing and treating skeletal muscle wasting.

\section{References}

Alway SE, Siu PM. Nuclear apoptosis contributes to sarcopenia. Exerc Sport Sci Rev 2008; 36:51-7.

Ansved T, Larsson L. Effects of ageing on enzyme-histochemical, morphometrical and contractile properties of the soleus muscle in the rat. J Neurol Sci 1989;93:105-24.

Attaix D, Mosoni L, Dardevet D, Combaret L, Patureau Mirand P, Grizard J. Altered responses in skeletal muscle protein turnover during aging in anabolic and catabolic periods. Int J Biochem Cell Biol 2005; 37:1962-73.

Bernhard W. A new staining procedure for electron microscopic cytology. J Ultrastruct Res 1969;27:250-65.

Biggiogera M, Bottone MG, Scovassi AI, Soldani C, Vecchio $L$, Pellicciari C. Rearrangement of nuclear ribonucleoprotein (RNP) containing structures during apoptosis and transcriptional arrest. Biol Cell 2004; 96: 603-15.

Biggiogera M, Cisterna B, Spedito A, Vecchio L, Malatesta M. Perichromatin fibrils as early markers of transcriptional alterations. Differentiation 2007; 76:57-65.

Borisov AB, Carlson BM. Cell death in denervated skeletal muscle is distinct from classical apoptosis. Anat Rec 2000; 258:305-18.

Brooks NE, Schuenke MD, Hikida RS. Ageing influences myonuclear domain size differently in fast and slow skeletal muscle of rats. Acta Physiologica 2009; doi: 10.1111/j.1748-1716.2009.01983.x.

Bruunsgaard $\mathrm{H}$, Pedersen M, Pedersen BK. Aging and proinflammatory cytokines. Curr Opin Hematol 2001;8:131-6.

Bruusgaard JC, Liestøl K, Gundersen K. Distribution of myonuclei and microtubules in live muscle fibers of young, middle-aged, and old mice. J Appl Physiol 2006; 100:2024-30.

Cardani R, Mancinelli E, Rotondo G, Sansone V, Meola G. Muscleblindlike protein 1 nuclear sequestration is a molecular pathology marker of DM1 and DM2. Eur J Histochem 2006;50:177-82.

Cisterna B, Flach F, Vecchio L, Barabino SML, Battistelli S, Martin TE, et al. Can a GMO-containing diet influence embryo development? A preliminary study on pre-implantation mouse embryos. Eur $\mathrm{J}$ Histochem 2008;52:263-7.

Combaret L, Dardevet D, Béchet D, Taillandier D, Mosoni L, Attaix D. Skeletal muscle proteolysis in aging. Curr Opin Clin Nutr Metab Care 2009:12:37-41.

Deschenes MR. Effects of aging on muscle fibre type and size. Sports Med 2004; 34:809-24.

Dirks $A$, Leeuwenburgh $C$. The role of apoptosis in age-related skeletal muscle atrophy. Sports Med 2005;35:473-83.

Doherty TJ. Invited review: Aging and sarcopenia. J Appl Physiol 2003;95:1717-27.
Dupont-Versteegden EE. Apoptosis in muscle atrophy: relevance to sarcopenia. Exp Gerontol 2005; 40:473-81.

Edström E, Ulfhake B. Sarcopenia is not due to lack of regenerative drive in senescent skeletal muscle. Aging Cell 2005;4:65-77.

Fakan S. Ultrastructural cytochemical analyses of nuclear functional architecture. Eur J Histochem 2004; 48:5-14.

Gallegly JC, Turesky NA, Strotman BA, Gurley CM, Peterson CA, Dupont-Versteegden EE. Satellite cell regulation of muscle mass is altered at old age. J Appl Physiol 2004; 97: 1082-90.

Gundersen K, Bruusgaard JC. Nuclear domains during muscle atrophy: nuclei lost or paradigm lost? J Physiol 2008; 586.11: 2675-81.

Hallen A. Accumulation of insoluble protein and ageing. Biogerontol 2002;3:307-15

Hepple RT. Sarcopenia: a critical perspective. Sci Aging Knowledge Environ 2003; 2003:pe31.

Hughes VA, Frontera WR, Roubenoff $R$, Evans $W J$, Singh MA. Longitudinal changes in body composition in older men and women: role of body weight change and physical activity. Am J Clin Nutr 2002; 76:473-81.

Jameson CW. Towards a unified and interdisciplinary model of ageing. Med Hypotheses 2004;63:83-6.

Kadi F, Charifi N, Denis C, Lexell J. Satellite cells and myonuclei in young and elderly women and men. Muscle Nerve 2004:29:120-7.

Karakelides H, Sreekumaran Nair K. Sarcopenia of aging and its metabolic impact. Curr Top Dev Biol 2005;68:123-48.

Kim JH, Choy HE, Nam KH, Park SC. Transglutaminase-mediated cross-linking of specific core histone subunits and cellular senescence. Ann N Y Acad Sci 2001;928:65-70.

Koopman R, van Loon LJC. Aging, exercise and muscle protein metabolism. J Appl Physiol (January 8, 2009). doi:10.1152/japplphysiol.91551.2008.

Lafarga M, Berciano MT, Andres MA. Protein-synthesis inhibition induces perichromatin granule accumulation and intranuclear rodlet formation in osmotically stimulated supraoptic neurons. Anat Embryol 1993;187:363-9.

Larsson L, Sjodin B, Karlsson J. Histochemical and biochemical changes in human skeletal muscle with age in sedentary males, ages 22-65 years. Acta Physiol Scand 1978;103:31-9.

Leeuwenburgh C. Role of apoptosis in sarcopenia. J Gerontol 2003; 58A: 999-1001.

Lesnefsky EJ, Hoppel CL. Oxidative phosphorylation and aging. Ageing Res Rev 2006;5:402-33.

Lexell J. Human aging, muscle mass, and fibre type composition. $J$ Gerontol A Biol Sci Med Sci 1995;50:11-6.

Lührmann R, Kastner B, Bach M. Structure of spliceosomal snRNPs and their role in pre-mRNA splicing. Biochim Biophys Acta 1990; 1087:265-92.

Malatesta M, Bertoni-Freddari C, Fattoretti P, Baldelli B, Fakan S, Gazzanelli G. Aging and vitamin E deficiency are responsible for altered RNA pathways. Ann N Y Acad Sci 2004; 1019:379-82.

Malatesta M, Bertoni-Freddari C, Fattoretti P, Caporaloni C, Fakan S, Gazzanelli G. Altered RNA structural constituents in aging and vitamin E deficiency. Mech Ageing Dev 2003;124:175-81.

Malatesta M, Perdoni F, Battistelli S, Muller S, Zancanaro C. The cell nuclei of skeletal muscle cells are transcriptionally active in hibernating edible dormice. BMC Cell Biol 2009;10:19.

Malatesta M, Perdoni F, Santin G, Battistelli S, Muller S, Biggiogera M. Hepatoma tissue culture (HTC) cells as a model for investigating the effects of low concentrations of herbicide on cell structure and function. Toxicol in vitro $2008 ; 22: 1853-60$.

Manta P, Vassilopoulos D, Spengos M. Nucleo-cytoplasmic ratio in ageing skeletal muscle. Eur Arch Psychiatry Neurol Sci 1987; 236:235-6.

Marbaix G, Huez G, Burny A, Cleuter Y, Hubert E, Leclercq M, et al. Absence of polyadenylate segment in globin messenger RNA accelerates its degradation in Xenopus oocytes. Proc Natl Acad Sci USA 1975;72:3065-7.

Payne GW, Bearden SE. The microcirculation of skeletal muscle in aging. Microcirculation 2006; 13:275-7.

Pujol G, Soderqvist H, Radu A. Age-associated reduction of nuclear protein import in human fibroblast. Biochem Biophys Res Comm $2002 ; 274: 354-8$. 
Puvion E, Puvion-Dutilleul F. Ultrastructure of the nucleus in relation to transcription and splicing: roles of perichromatin fibrils and interchromatin granules. Exp Cell Res 1996; 229:217-25.

Puvion E, Viron A, Bernhard W. Unusual accumulation of ribonucleoprotein constituents in the nucleus of cultured rat liver cells after hypothermal shock. Biol Cell 1977; 29:81-8.

Puvion-Dutilleul F, Puvion E. Relationship between chromatin and perichromatin granules in cadmium-treated isolated hepatocytes. J. Ultrastruct. Res. 1981; 7:341-50.

Rodrigues Ade C, Schmalbruch H. Satellite cells and myonuclei in long-term denervated rat muscles. Anat Rec 1995;243:430-7.

Roijkind M, Dominguez-Rosales JA, Nieto N, Greenweld P. Role of hydrogen peroxide and oxidative stress in healing responses. Cell Mol Life Sci 2002; 59:1872-91.

Roubenoff R. Sarcopenia and its implications for the elderly. Eur J Clin Nutr 2000; 54:S40-7.

Ryall JG, Schertzer JD, Lynch GS. Cellular and molecular mechanisms underlying age-related skeletal muscle wasting and weakness. Biogerontol 2008;9:213-28.

Schwarzacher HG, Wachtler F. The nucleolus. Anat Embryol 1993; 188:515-36.

Shaw PJ, Jordan EG. The nucleolus. Ann Rev Cell Dev Biol 1995; 11:93-121.

Shorey CD, Manning LA, Everitt AV. Morphometrical analysis of skeletal muscle fibre ageing and the effect of hypophysectomy and food restriction in the rat. Gerontol 1988; 34:97-109.

Soreq $H$, Nudel $U$, Salomon R, Revel M, Littauer UZ. In vitro translation of polyadenylic acid-free rabbit globin messenger RNA.J Mol Biol 1974;88:233-45.

Spector DL. Nuclear organization and gene expression. Exp Cell Res 1996;229:189-97.

Stadtman ER. Protein oxidation and aging. Free Radic Res 2006; 40:1250-8.

Stadtman ER. Role of oxidant species in aging. Curr Med Chem 2004; 11:1105-12.
Szulc P, Beck TJ, Marchand F, Delmas PD. Low skeletal muscle mass is associated with poor structural parameters of bone and impaired balance in elderly men: the MINOS study. J Bone Miner Res 2005; 20:721-9.

Tenover JL. Testosterone and the aging male. J Androl 1997;18:103-6. Testillano PS, Gorab E, Risueno MC. A new approach to map transcription sites at the ultrastructural level. J Histochem Cytochem $1994 ; 42: 1-10$.

Thompson LD. Age-related muscle dysfunction. Exp Gerontol 2009; 44:106-11.

Tomonaga M. Histochemical and ultrastructural changes in senile human skeletal muscle. J Am Geriatr Soc 1977; 25:125-31.

Veraldi KL, Arhin GK, Martincic K, Chung-Ganster LH, Wilusz J, Milcarek C. HnRNP F influences binding of a 64-kilodalton subunit of cleavage stimulation factor to mRNA precursors in mouse $B$ cells. Mol Cell Biol 2001; 21:1228-38.

Verdijk LB, Koopman R, Schaart G, Meijer K, Savelberg HH, van Loon LJ. Satellite cell content is specifically reduced in type II skeletal muscle fibers in the elderly. Am J Physiol Endocrinol Metab 2007; 292:E151-7.

Wada KI, Katsuta S, Soya H. Natural occurrence of myofiber cytoplasmic enlargement accompanied by decrease in myonuclear number. Japanese J Physiol 2003; 53:145-50.

Wheeler TM, Thornton CA. Myotonic dystrophy: RNA-mediated muscle disease. Curr Opin Neurol 2007; 20:572-6.

Wilson MM, Morley JE. Invited review: Aging and energy balance. J Appl Physiol 2003;95:1728-36.

Zancanaro C, Malatesta M, Vogel $P$, Osculati F, Fakan S. Ultrastructural and morphomentrical analyses of the brown adipocyte nucleus in a hibernating dormouse. Biol Cell 1993;79:5561.

Zancanaro C, Mariotti R, Perdoni F, Nicolato E, Malatesta M. Physical training is associated with changes in NMR and morphometrical parameters of the skeletal muscle in senescent mice. Eur $J$ Histochem 2007; 51: 305-10. 